\title{
De novo transcriptome assembly for the five major organs of Zanthoxylum armatum and the identification of genes involved in terpenoid compound and fatty acid metabolism
}

Wen-Kai Hui ${ }^{1}$, Fei-Yan Zhao ${ }^{1}$, Jing-Yan Wang ${ }^{1}$, Xiao-Yang Chen ${ }^{2 *}$ (D, Jue-Wei Li ${ }^{1}$, Yu Zhong ${ }^{1}$, Hong-Yun Li ${ }^{3}$, Jun-Xing Zheng ${ }^{1}$, Liang-Zhen Zhang ${ }^{1}$, Qing-Min Que ${ }^{2}$, Ai-Min Wü ${ }^{2^{*}}$ and Wei Gong ${ }^{1^{*}}$

\begin{abstract}
Background: Zanthoxylum armatum (Z. armatum) is a highly economically important tree that presents a special numbing taste. However, the underlying regulatory mechanism of the numbing taste remains poorly understood. Thus, the elucidation of the key genes associated with numbing taste biosynthesis pathways is critical for providing genetic information on $Z$. armatumand the breeding of high-quality germplasms of this species.

Results: Here, de novo transcriptome assembly was performed for the five major organs of $Z$. armatum, including the roots, stems, leaf buds, mature leaves and fruits. A total of 111,318 unigenes were generated with an average length of $1014 \mathrm{bp}$. Additionally, a large number of SSRs were obtained to improve our understanding of the phylogeny and genetics of $Z$. armatum. The organ-specific unigenes of the five major samples were screened and annotated via GO and KEGG enrichment analysis. A total of 53 and 34 unigenes that were exclusively upregulated in fruit samples were identified as candidate unigenes for terpenoid biosynthesis or fatty acid biosynthesis, elongation and degradation pathways, respectively. Moreover, 40 days after fertilization (Fr4 stage) could be an important period for the accumulation of terpenoid compounds during the fruit development and maturation of $Z$. armatum. The Fr4 stage could be a key point at which the first few steps of the fatty acid biosynthesis process are promoted, and the catalysis of subsequent reactions could be significantly induced at 62 days after fertilization (Fr6 stage).

Conclusions: The present study realized de novo transcriptome assembly for the five major organs of $Z$. armatum. To the best of our knowledge, this study provides the first comprehensive analysis revealing the genes underlying the special numbing taste of $Z$. armatum. The assembled transcriptome profiles expand the available genetic information on this species and will contribute to gene functional studies, which will aid in the engineering of high-quality cultivars of $Z$. armatum.
\end{abstract}

Keywords: Zanthoxylum armatum, De novo transcriptome, Aromatic compounds, Fatty acid

\footnotetext{
*Correspondence: xychen_bjfu@163.com; wuaimin@scau.edu.cn; gongwei@sicau.edu.cn

${ }^{2}$ Guangdong Key Laboratory for Innovative Development and Utilization of

Forest Plant Germplasm, State Key Laboratory for Conservation and

Utilization of Subtropical Agro-Bioresources, College of Forestry and

Landscape Architecture, South China Agricultural University, Guangzhou

510642, China

'Key Laboratory of Ecological Forestry Engineering of Sichuan Province,

College of Forestry, Sichuan Agricultural University, Chengdu 611130, China

Full list of author information is available at the end of the article
}

(c) The Author(s). 2020 Open Access This article is distributed under the terms of the Creative Commons Attribution 4.0 International License (http://creativecommons.org/licenses/by/4.0/), which permits unrestricted use, distribution, and reproduction in any medium, provided you give appropriate credit to the original author(s) and the source, provide a link to the Creative Commons license, and indicate if changes were made. The Creative Commons Public Domain Dedication waiver (http://creativecommons.org/publicdomain/zero/1.0/) applies to the data made available in this article, unless otherwise stated. 


\section{Background}

Zanthoxylum armatum (Rutaceae), commonly known as green Sichuan pepper, is one of the most economically important trees in Sichuan Province and is widely distributed in most parts of southwest China and some parts of southeast Asia [1]. The production of Z. armatum is currently a billions of dollars commercially, and this species has a long history of cultivation in China because it is one of the eight main spices used in Chinese cuisine and is an essential ingredient in Sichuan hot-pot, with a special numbing taste [2]. However, the underlying regulatory mechanism of the genes associated with the numbing taste remains poorly understood. Z. armatum is also an important medicinal plant prioritized by the governments of some countries for economic development [3-5]. Its fruit berries can be used for the treatment of abdominal pain, rheumatism, and skin diseases, and as a carminative and antispasmodic [3]. The seeds are employed as an aromatic tonic for conditions such as fever, dyspepsia, toothache, and stomach ache [3, 4]. Thus, the elucidation of the keygenes associated with numbing taste biosynthesis pathways, especially those responsible for the accumulation of the main compounds involved, is critical for revealing genetic information for this species and breeding highquality $Z$. armatum germplasms.

In previous studies, the numbing taste was found to be associated with the presence of volatile oils, alkaloids, coumarins, acid amide phenol components and so on $[6,7]$, which accumulate at high levels in the fruits, leaves, stems, and roots of $Z$. armatum, especially in the pericarps [8]. More than 140 components related to aromatic compounds and fatty acid biosynthesis have been gradually identified in different tissues of $Z$. armatum [9]. Various terpenoid substances are among the main components associated with the numbing taste of $Z$. armatum, including linalool (29.30\%), limonene (14.30\%), myrcene (6.02\%), cineole $(1.32 \%)$ and so on $[7,10]$. Terpenoids are a large category of necessary secondary metabolites in plants, includingmonoterpenes, diterpenes, sesquiterpenes, triterpenoids and other terpenoid-quinone compounds [11]. All of these downstream products accumulate from the terpenoid backbone biosynthesis pathway. The five key enzymes, acetoacetyl-CoA thiolase 2 (ACAT2), hydroxymethyl-glutaryl-CoA synthase (HMGS), hydroxy-methylglutaryl-CoA reductase (HMGR), mevalonate kinase (MVK), and diphosphomevalonate decarboxylase (MVD) are required for the only pathway producing isopentenylPP upstream of terpenoid backbone biosynthesis [12]. Subsequently, the production of dimethylallyl- $P P$ from isopentenyl- $P P$ can be catalysed by isopentenyl-diphosphate deltaisomerase (IDI) [13]. Additionally, dimethylallyl-PP can be produced via the methylerythritol phosphate (MEP) pathway. Next, isopentenyl- $P P$ and dimethylallyl- $P P$ are transformed into farnesyl- $P P$ and geranyl-geranyl- $P P$, which are then used to produce various terpenoids, catalysed by different diphosphate synthases [14]. Fatty acids are also important productsfound in the fruit of $Z$. armatum. Abundant unsaturated fatty acids have been detected in the seeds of Zanthoxylum (approximately 25\%), among which linolenic acid, linoleic acid and oleic acid account for $78.63 \%$ (mass percentage) of the total, while the main saturated fatty acid is hexadecanoic acid $(13.18 \%)[15,16]$. The production of fatty acids is involved in the de novo fatty acid biosynthesis, elongation and degradation pathways [16]. In the de novo fatty acid biosynthesis pathways, acetyl-CoA is produced and used to form malonyl-ACP, catalysed by acetyl-CoA carboxylase (ACC) and malonyl-transferase (MCMT). Subsequently, malonyl-ACP can be converted to a long-chain acyl-ACP catalysed by several enzymes with an acyl carrier protein (ACP) as a co-factor [17]. Three main enzymes that play an important role during this series of reactions include ketoacyl-ACP synthase II (KASII), ketoacyl-ACP synthase I (KASI), and oxoacyl-acyl-carrier protein reductase (FabG) [18]. Moreover, ACP desaturase 5 (AAD5), also known asstearoyl-ACP desaturase (SAD), removes two hydrogen atoms from stearic acid to form oleic acid in unsaturated fatty acid production [19]. Finally, $N$-acyl-ACP (C8 to C18) is hydrolysed to release free fatty acids [20]. Thus far, many studies on Zanthoxylum have mainly dealt with plant breeding, physiological investigations, tissue culture and chemical component identification and extraction in the fruits $[8,21]$. A recent study identified the key genes in the synthesis pathway of volatile terpenoids in the fruit of Zanthoxylum bungeanum (red Sichuan pepper) [22]. However, the crucial genes involved in the biosynthesis of terpenoid compounds and fatty acids have not been reported in $Z$. armatum.

Comparative transcriptome analysis is an important method for rapidly obtaining a large amount of genetic information and putative candidate genes related to target traits by examining different tissue samples. Although transcriptome sequencing analyses have rarely been reported in $Z$. armatum, they have been conducted frequently in other tree species. The de novo transcriptome sequencing of eight major organs of Plukenetia volubilis was performed to identify candidate genes involved in $\alpha$-linolenic acid metabolism, thereby expanding the genetic information available for functional genome studies of $P$. volubilis [20]. Through the transcriptome sequencing analysis of roots, stems, leaves, arils and kernel samples of two Torreya grandis cultivars, six candidate unigenes encoding sciadonic acid elongase and desaturases were identified to improve the understanding of the molecular mechanisms responsible for de novo fatty acid biosynthesis in gymnosperm species [16]. Additionally, Wang et al. [21] conducted a detailed transcriptional sequencing analysis of two orange varieties at different fruit development stages to elucidate 
the underlying regulatory mechanism of sucrose and citrate accumulation in the ripening of the fruits, especially during the fruit delayed-harvest stage. Zhang et al. [23] explored the key regulatory factors involved in starch and sucrose metabolism in Castanea mollissima via transcriptome sequencing of seeds at various developmental stages. However, only a single study has reported transcriptome profiles obtained using a mixture of the leaves and inflorescences of $Z$. armatum to isolate the viruses associated with flower yellowing disease in recent years [24].

In the present study, the de novo transcriptome sequencing of five major organs was performed using the Illumina HiSeq 4000 platform. The key candidate genes associated with terpenoid compounds and fatty acid biosynthesis and metabolism were identified from the RNA-seq dataset in $Z$. armatum. Furthermore, samples from different stages in the fruit development and maturation process were selected to identify the expression patterns of the key candidate genes through qRT-PCR analysis. To the best of our knowledge, ours is first comprehensive analysis to reveal the genes underlying the special numbing taste of $Z$. armatum. The assembled transcriptome profiles expand the available genetic information for $Z$. armatum and provide an improved understanding for gene function studies, which will aid in the engineering of high-quality varieties of $Z$. armatum.

\section{Results}

Transcriptome sequencing and de novo assembly of $Z$. armatum

The total RNA of five major sample types, including roots (Ro), stems (St), leaf buds (LB), mature leaves (ML) and fruits (Fr), was isolated to construct the comprehensive transcriptome of $Z$. armatum. The quality of the RNA was determined using the OD260/OD280 ratio (1.88-2.21) and RIN (7.60-10.00) (Additional file 1: Table S1). A total of $126.89 \mathrm{G}$ of paired-end raw reads were produced by transcriptome sequencing. After trimming adapters and low-quality bases, 7.92-9.91 G of clean bases were produced from 15 cDNA libraries in this study (Additional file 2: Table S2). The error rate of RNA-seq was only approximately $0.02 \%$, all of the Q30 values were greater than $93.90 \%$, and the GC content was above $43 \%$ in each sample. In addition, an assembly of 350,625 transcripts was achieved, with a mean length of $1219 \mathrm{bp}$ for the $Z$. armatum transcriptome (Additional file 3: Figure S1a). The longest transcript of each gene was chosen from the assembly results as the candidate unigene. Finally, a set of 111,318 unigenes was obtained in the present study (Additional file 3: Figure S1b). The length of the unigenes ranged from 301 to $17,299 \mathrm{bp}$, with an average of $1014 \mathrm{bp}$, and the lengths of more than half of the unigenes in the total assembly were greater than 1454 bp $(N 50=1454)$.

\section{Identification of simple sequence repeats}

In recent years, various molecular markers have been widely developed in different plants to construct plant genetic maps, perform gene localization, determine hybrid purity, and examine other aspects. To obtain abundant molecular markers for the genetic analysis and marker-assisted selection breeding of Z. armatum, simple sequence repeats (SSRs) were identified in our transcriptome (Fig. 1). Mono- to hexanucleotide SSRs were identified using MISA software (Fig. 1, Additional file 4: Table S3). A total of 46,098 SSR loci were characterized, among which mononucleotide repeats were the most abundant (30,266, 65.66\%), followed by dinucleotides (8528, 18.50\%). Moreover, at least 9-12 repeats were detected among the monomer nucleotide SSRs. However, di- to hexanucleotide SSRs were explored mostly in the context of 5 to 8 repeats. These results indicated that high variation might exist in $Z$. armatum.

\section{Gene functional annotation}

After assembly, all the unigenes were subjected to BLASTx analysis against five public databases, including NR, NT, KO, Pfam, and KOG, to characterize their gene functions (Additional file 5: Figure S2, Table 1). A total of $73,426(65.96 \%)$ unigenes were annotated in at least one database. The highest annotation rate was obtained in the NR database, which assigned 61,598 (55.34\%) unigenes. A total of 34,930 (31.38\%) and 16,316 (14.66\%) unigenes were annotated with the Pfam and KOG databases, respectively. The top-scoring BLASTx hits against the NR protein database showed that the E-value distribution presented a comparable pattern with $50.70 \%$ of the mapped sequences with high homologies $(<1 \mathrm{e}-45)$, whereas the E-values for $49.30 \%$ of the homologous sequences ranged between $1 \mathrm{e}-5$ and $1 \mathrm{e}-45$ (Additional file 5: Figure S2a). The distribution of sequence similarity showed that $53.90 \%$ of the mapped sequences presented similarities higher than $80 \%$, while $10.50 \%$ of the hits exhibited similarities lower than 60\% (Additional file 5: Figure S2b). Additionally, the species distribution of the NR BLASTx matches showed that the top three species were Citrus clementina (21.80\%), Citrus unshiu (19.00\%) and Citrus sinensis (16.10\%), and all of these species and $Z$. armatum belonged to Rutaceae (Additional file 5: Figure S2d). All the above results indicated that a highquality annotation was obtained in the present study.

Moreover, 34,930 (31.37\%) unigenes were annotated with 55 Gene Ontology (GO) terms, including 26 terms related to biological processes, 19 terms related to cellular components, and 10 terms associated with molecular functions (Additional file 6: Figure S3). Under the biological process, cellular component and molecular function categories, the predominant groups were assigned to the cellular process (GO: 0009987) and metabolic 


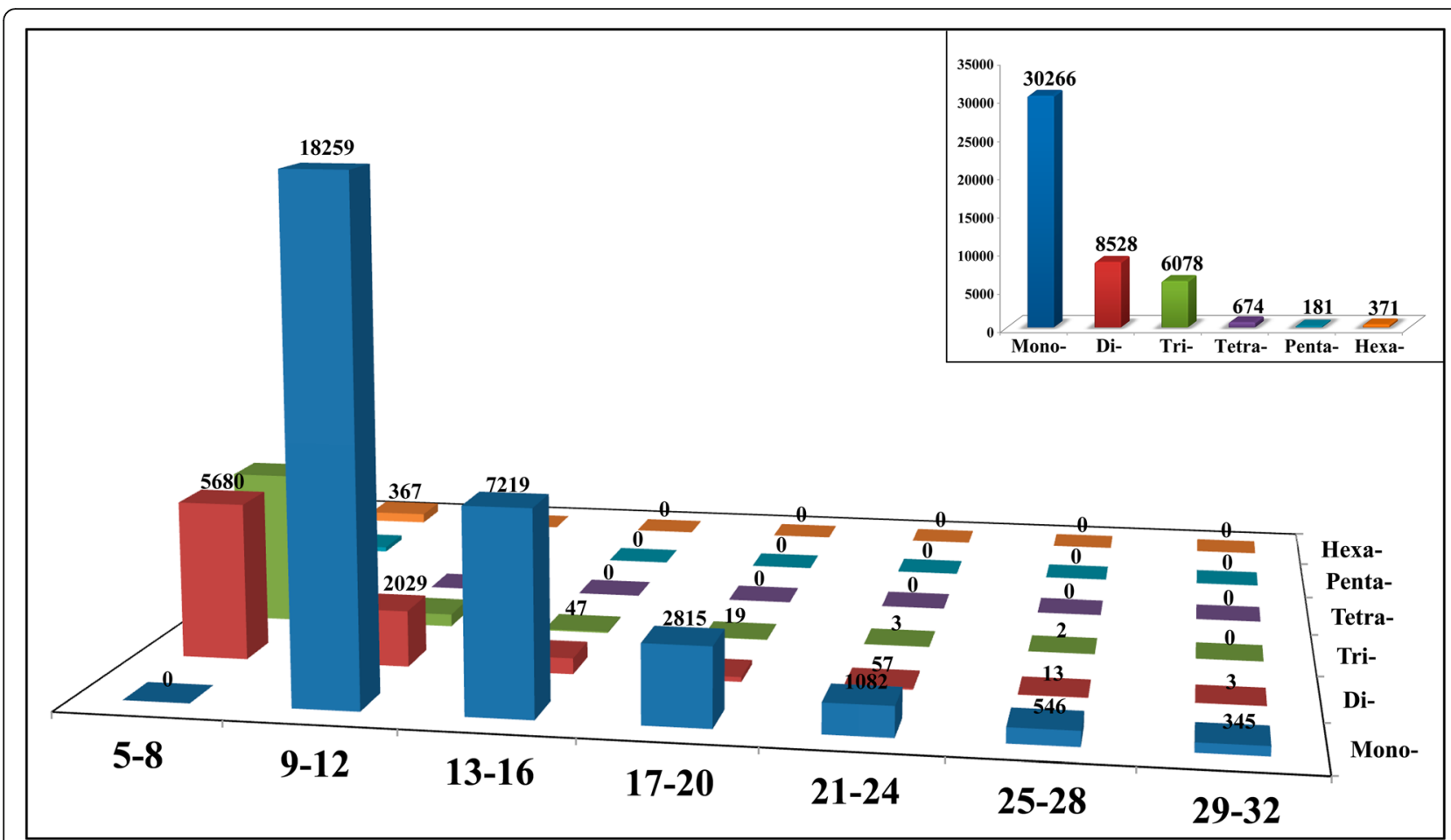

Fig. 1 The distribution of SSRs in Z. armatum. The insert shown the distribution of the total number of SSRs in mono-, di-, tri-, tetra-, penta- and hexa-nucleotide repeats

process (GO: 0008152); cell (GO: 0005623) and cell part (GO: 0044464); and binding (GO: 0005488) and catalytic activity (GO: 0003824) terms, respectively. To further understand the biological functions and interactions of the unigenes, they were also classified into metabolic pathways using the Kyoto Encyclopedia of Genes and Genomes (KEGG). A total of 24,137 unigenes (21.68\%) were assigned to 19 categories divided into five clusters, including cellular processes, environmental information processing, genetic information processing, metabolism and organismal systems (Additional file 7: Figure S4). Among the KEGG pathways (Additional file 8: Table S4), the top five categories were translation (2916 unigenes),

Table 1 Summary of the annotation about the Zanthoxylum armatum transcriptome

\begin{tabular}{lll}
\hline Items & $\begin{array}{l}\text { Number of } \\
\text { Unigenes }\end{array}$ & Percentage (\%) \\
\hline Annotated in NR & 61,598 & 55.34 \\
Annotated in NT & 55,465 & 49.83 \\
Annotated in KO & 24,137 & 21.68 \\
Annotated in Pfam & 34,930 & 31.38 \\
Annotated in KOG & 16,316 & 14.66 \\
Annotated in all Databases & 4656 & 4.18 \\
Annotated in at least one Database & 73,426 & 65.96 \\
Total Unigenes & 111,318 & 100 \\
\hline
\end{tabular}

carbohydrate metabolism (2226 unigenes), overview (1771 unigenes), folding and degradation (1659 unigenes), and amino acid metabolism (1394 unigenes). All of these results showed that the investigated samples were characterized by active cell development and differentiation. Four hundred and twenty two unigenes were annotated with metabolism of terpenoids and polyketides, and 376 of which were related to monoterpenoid, diterpenoid, sesquiterpenoid and triterpenoid, limonene and pinene, carotenoid, and terpenoid backbone biosynthesis pathways. Additionally, 314 unigenes were involved in fatty acid biosynthesis and metabolism. These unigenes were the main substances associated with fatty acid and aromatic compound accumulation. It is worth noting that all of these putative genes were differentially expressed among the five different samples in this study (Additional file 9: Figure S5, Additional file 10: Figure S6), and more unigenes were up-regulation in the fruit samples. This suggested that some organ-specific unigenes might be existed in various tissues, and the fruit could be the mainly organs to accumulate the numbing-taste related compounds.

\section{Investigation of organ-specific unigenes}

The Pearson correlation analysis revealed that all three independent biological replicates of each sample presented good reproducibility in the present study, and the 
stem samples showed the highest correlation coefficient among all of the investigated organs (Additional file 11: Figure S7). To further analyse the characteristics of the genes related to the different organs, the organ-specific unigenes of the five major samples were screened on the basis of a $p$ value $<0.05$ and $\mid \log 2$ (fold change) $\mid>5$. The expression values (FPKM) for each comparison were for one organ and the sum of other organs. The investigation of the organ-specific unigenes expressed in each organ showed that 4970, 90, 2314, 1955, and 650 unigenes were specifically found in the roots, stems, leaf buds, mature leaves, and fruits, respectively (Additional file 12: Figure S8a-e). The root samples (Ro) expressed the most unigenes (49.80\%), including 2653 upregulated and 2317 downregulated genes (Additional file 12: Figure S8f). The stems and fruits expressed the fewest unigenes $(0.90$ and $6.51 \%$, respectively). To evaluate the functional properties of these organ-specific unigenes, KEGG enrichment was performed, and the significant pathways of each organ are listed in (Additional file 13: Table S5). Seventeen KEGG pathways were significantly enriched in root samples, including flavonoid biosynthesis, phenylpropanoid biosynthesis, terpenoid biosynthesis, alkaloid biosynthesis, and zeatin biosynthesis, which indicated that the most active biological synthesis was occurring in the roots of $Z$. armatum. Only two significant KEGG pathways (five unigenes) were observed in the stem samples. Additionally, 14 and 16 KEGG pathways were distinctively obtained in the LB and ML samples, respectively. In both cases, the greatest enrichment was observed for phenyl propanoid biosynthesis, glycerolipid metabolism, galactose metabolism, pentose and glucuronate interconversions, glycosphingolipid biosynthesis, and glycolysis and gluconeogenesis pathways, which supply the necessary substances for the growth and development of these tissues. However, unigenes involved in plant hormone signal transduction, flavonoid and terpenoid biosynthesis and metabolism were detected at significant levels in ML samples, which might contribute to the numbing taste and peppery flavour of the leaves of $Z$. armatum. Additionally, 18 significant KEGG pathways were identified in the Fr samples, most of which were enriched in terpenoid, alkaloid and flavonoid biosynthesis. These unigenes might be the main source of the special numbing taste of the fruits of $Z$. armatum.

\section{Identification and characterization of genes involved in terpenoid biosynthesis}

The analysis of differentially expressed genes (DEGs) was carried out in the five major organs of $Z$. armatum. To comprehensively reveal the key genes associated with the special numbing taste in the fruits of $Z$. armatum, genes with a $p$ value $<0.05$ and a $\mid \log 2$ (fold change) $\mid>1$ identified by EdgeR were regarded as DEGs in the comparisons of fruit samples and other organs ( $\mathrm{Fr}$ vs. $\mathrm{Ro}, \mathrm{Fr}$ vs. St, Fr vs. LB, and Fr vs. ML). As a result, a total of 3091 DEGs were co-detected in all four comparisons (Fig. 2a), 1625 of which were co-screened and found to be upregulated in all four comparisons (Fig. 2b), whereas 251 of which were co-identified and found to be downregulated in all four comparisons (Fig. 2c). The KEGG enrichment analysis showed that the downregulated DEGs were mainly related to plant hormone signal transduction and amino and sugar metabolism pathways (Additional file 14: Table S6). However, most of the upregulated DEGs were significantly enriched in terpenoid, alkaloid, flavonoid and fatty acid biosynthesis and

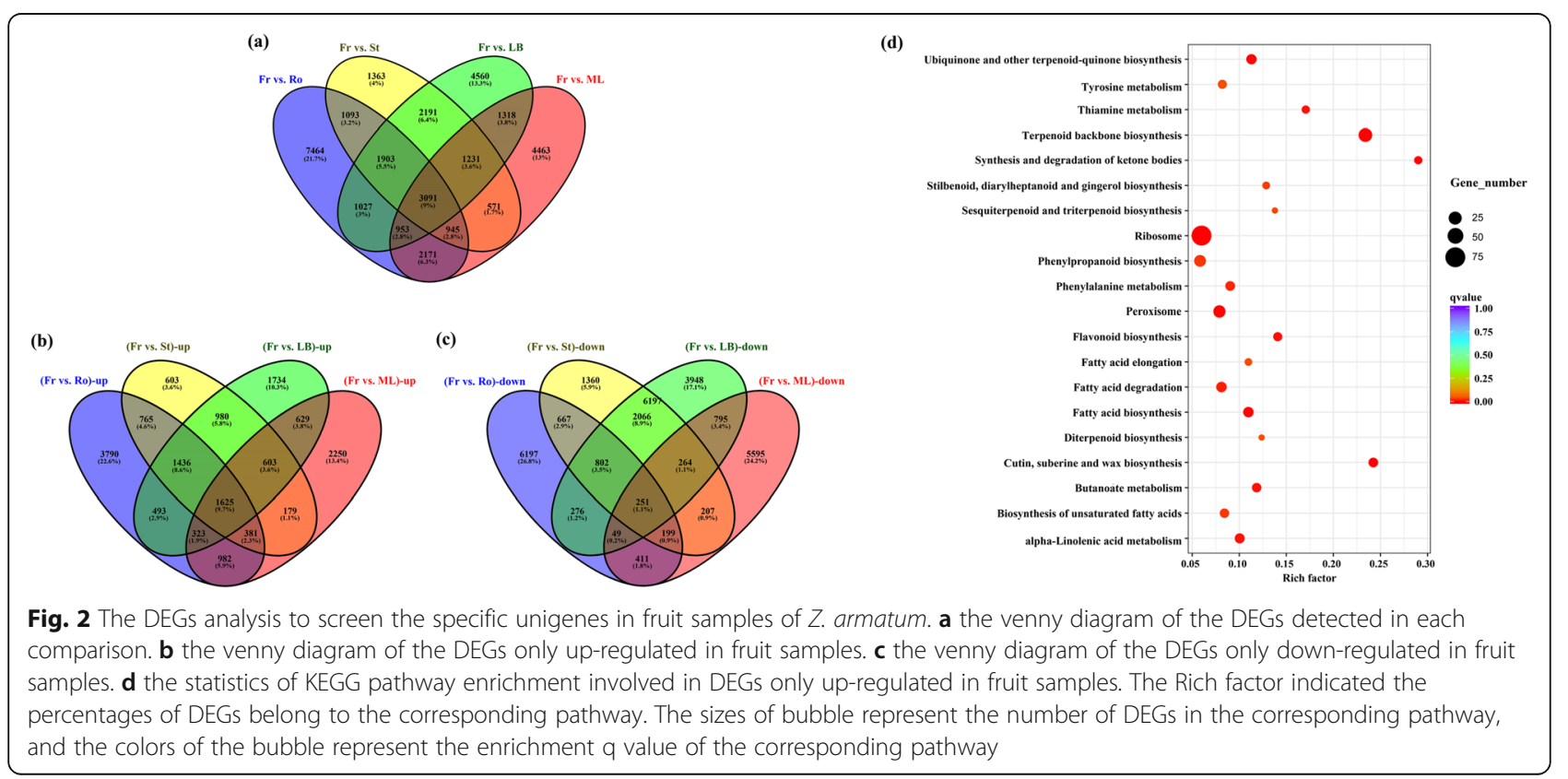


metabolism (Fig. 2d, Additional file 14: Table S6). Thus, the following analysis was mainly focused on the upregulated unigenes to explore the genetic information associated with the special numbing taste in $Z$. armatum.

In total, 53 DEGs were identified as candidate unigenes for 12 enzymes involved in terpenoid biosynthesis, and their expression values in the five major organs and TAIR10 annotations are shown in (Additional file 15: Table S7) and (Additional file 16: Table S8). These enzymes constituted two independent subpathways upstream of the terpenoid biosynthesis pathway (Fig. 3a), both of which utilize glycolysis to obtain the initial substrate for producing dimethylallyl diphosphate (dimethylallyl- $P P$ ). Then, dimethylallyl- $P P$ is used to generate monoterpenoids, diterpenoids, triterpenoids, and other terpenoid compounds [14], which are the main aromatic substances involved in the special numbing taste in the fruits of $Z$. armatum [10]. In the present study, the results showed that almost all genes involved in terpenoid backbone biosynthesis were differentially expressed genes and detected at significant levels in the fruit samples (Fig. 3a).
To further characterize the functional properties of these DEGs, some of the DEGs were selected to perform qRT-PCR detection during fruit development and maturation. A total of eight fruit samples were collected to investigate these genes (Additional file 17: Figure S10): in Fr1, 5 days after fertilization, the fruit was green-yellowish with a smooth surface; in Fr2, 15 days after fertilization, the fruit was oval with some slightly concave and transparent speckling on the surface; in Fr3, 28 days after fertilization, the fruit was green and grew rapidly; in Fr4, 40 days after fertilization, the fruit was further expanded with obvious speckles; in Fr5, 50 days after fertilization, the fruit gradually stopped expanding, and inclusions began to accumulate within the speckles; in Fr6, 62 days after fertilization, the fruit was dark green, and significant speckles accumulated additional inclusions; in Fr7, 75 days after fertilization, the fruit gradually matured and exhibited many inclusions within speckles; and in Fr8, 85 days after fertilization, the fruit was completely mature, and the special numbing taste was fully developed.

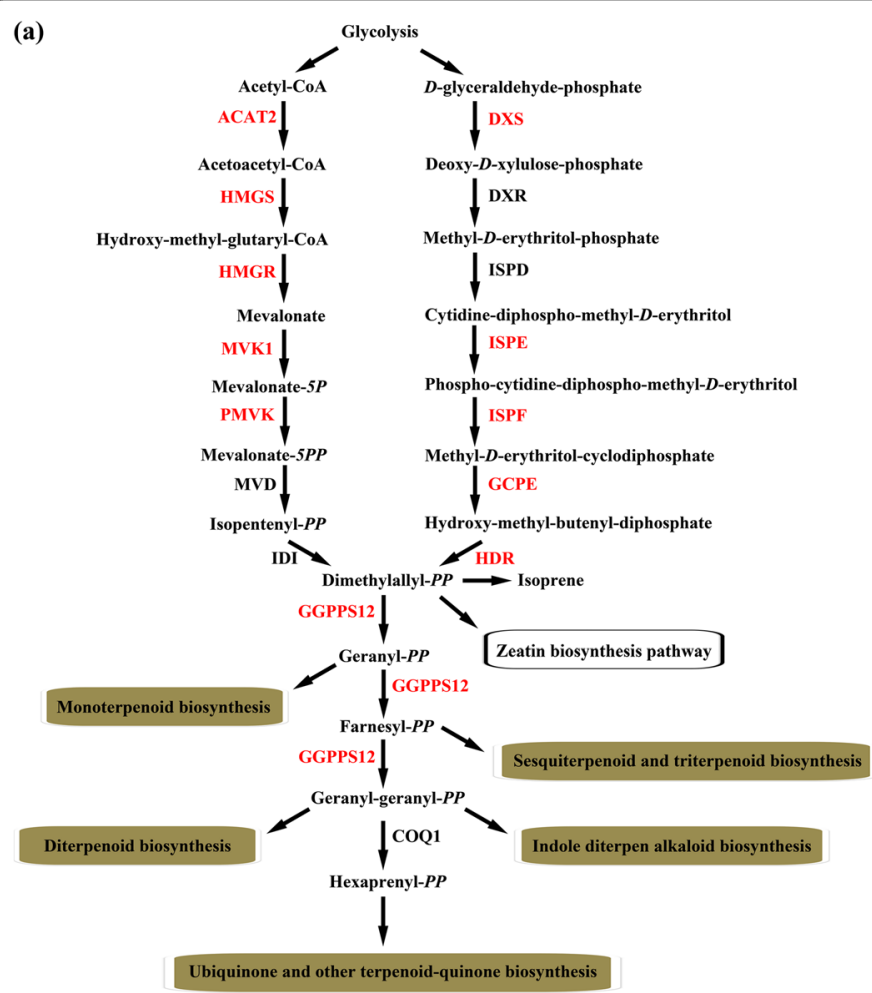

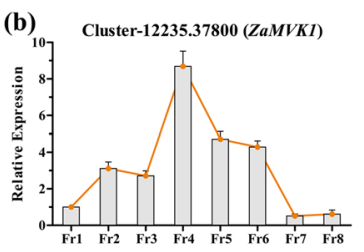
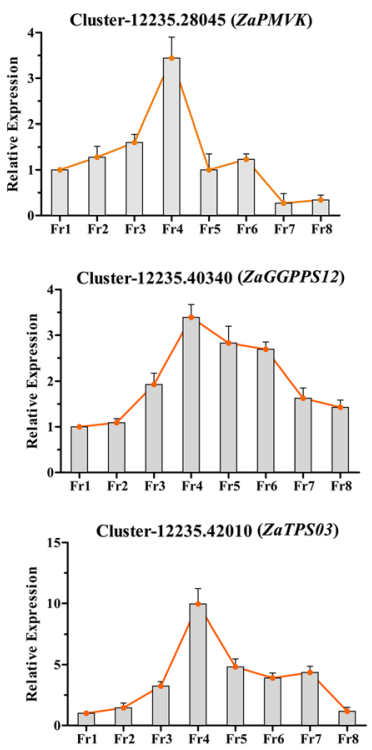
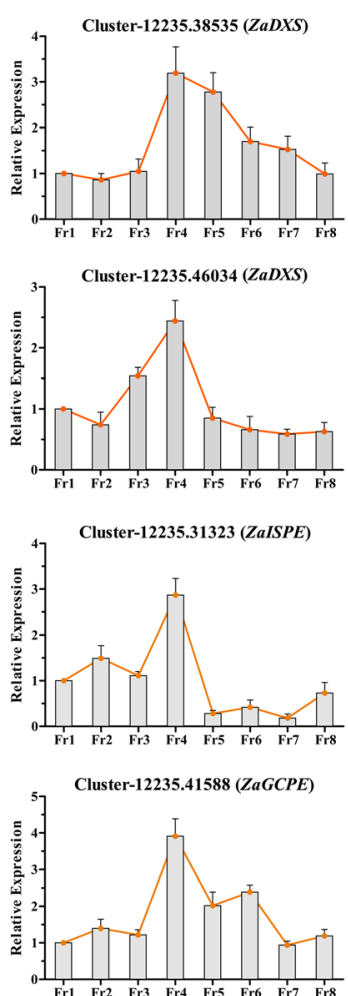

Fig. 3 The identification of genes in the pathway of terpenoid biosynthesis based on the transcriptome of $Z$. armatum. a the regulatory cascade of terpenoid biosynthesis pathway. Red fonts indicates the homologous differential expressed genes significantly up-regulated in this study and they were abbreviated as follows: ACAT2, Acetoacetyl-CoA Thiolase 2; HMGS, Hydroxy-methyl-glutaryl-CoA Synthase; HMGR, Hydroxy-methylglutaryl-CoA Reductase; MVK1, Mevalonate Kinase 1; PMVK, Phosphomevalonate Kinase; DXS, Deoxy-D-Xylulose 5-Phosphate Synthase; ISPE, Diphosphocytidyl-methyl-D-erythritol Kinase; ISPF, Isoprenoid F; GCPE, Hydroxy-methylbut-enyl Diphosphate Synthase; HDR, Hydroxy-methylbutenyl Diphosphate Reductase; GGPPS12, Geranylgeranyl diphosphate synthase 12. b the relative expression of the DEGs during fruit development and maturation process. The Fr1 was used as the control sample 
Two unigenes, Cluster-12,235.37800 and Cluster-12, 235.28045, were annotated to mevalonate kinase 1 $(M V K 1)$ and phosphomevalonate kinase $(P M V K)$, respectively, which belong to the MVA pathway and play rate-determining roles in the production of mevalonate-5PP. The results showed that both of these unigenes were significantly upregulated in the Fr4 stage but showed lower expression in the preceding and the subsequent stages of fruit development and maturation, especially in the Fr7 and Fr8 stages. Cluster-12, 235.38535 and Cluster-12,235.46034 were annotated to deoxy- $D$-xylulose phosphate synthase $(D X S)$, which catalyses the initial step in the transformation of $D$-glyceraldehyde-phosphate into deoxy- $D$-xylulose-phosphate in the MEP pathway. The results showed that both of these unigenes were significantly upregulated in the $\mathrm{Fr} 4$ stage but downregulated in the preceding and subsequent stages of fruit development and maturation (Fig. 3b). Additionally, unigenes involved in two enzymes in the MEP pathway, Cluster-12,235.31323 (ZaISPE) and Cluster-12, 235.41588 ( $Z a G C P E)$, presented similar expression patterns in the fruit development and maturation stages. Moreover, Cluster-12,235.40340, a key unigene annotated to geranyl-geranyl diphosphate synthase 12 (GGPPS12), which generates important substrates associated with the biosynthesis of various terpenoid compounds, presented gradual up-regulation from the Fr1 to Fr4 stages, whereas it was significantly downregulated in the Fr4 to Fr8 stages. A consistent result was that ZaTPSO3 (Cluster-12, 235.42010) was exclusively upregulated in Fr4 samples of $Z$. armatum; this unigene is related to the catalysis of the production of (R)-limonene as well as other related compounds using geranyl- $P P$ via the monoterpenoid biosynthesis process. These results indicated that the Fr4 stage could be the core period for the initiation of terpenoid compound biosynthesis and the accumulation of these compounds in the fruit development and maturation process of $Z$. armatum.

\section{Identification and characterization of genes involved in fatty acid biosynthesis}

Based on KEGG enrichment, a total of 20 DEGs were screened and annotated to fatty acid biosynthesis and elongation processes (Additional file 14: Table S6, Fig. 2d). The expression values of these candidate unigenes in the five major organs and their TAIR10 annotations are shown in (Additional file 15: Table S7) and (Additional file 16: Table S8). Moreover, all 20 DEGs were only associated with six subfamilies, including the ACP desaturase 5 (AAD5), acyl-activating enzyme 16 (AAE16), ketoacylACP synthetase II (KASII), long-chain acyl-CoA synthase (LACS), ketoacyl-CoA synthase (KCS), and oxoacyl-acylcarrier protein reductase $(F a b G)$ subfamilies, for which homologous Arabidopsis sequences were not identified in the TAIR10 protein database. Additionally, KASII, FabG, and $A A D 5$ were significantly involved in fatty acid biosynthesis pathways (Fig. 4a), whereas $L A C S$ and $K C S$ were mainly associated with long-chain fatty acid biosynthetic and metabolic processes (Additional file 16: Table S8).

Furthermore, ZaKASII (Cluster-12,235.36948), a key synthetase related to the elongation of 16-carbon palmitoylACP to produce 18-carbon stearoyl-ACP, was significantly upregulated in the Fr4 stage (Fig. 4b) but presented relatively low expression in the preceding and subsequent stages, especially in the fruit maturation process (Fr7-Fr8). However, Cluster-12,235.36948, annotatedto FabG, which reduces3-Oxo- $N$-ACP to form 3-hydroxy- $N$-ACP, was not only upregulated in the Fr4 stage but also showed higher expression in the Fr6-Fr8 stages. It is worth noting that two unigenes annotated to $Z a A A D 5$, Cluster-12,235.43479 and Cluster-12,235.42753, showed similar patterns and were exclusively upregulated in the Fr6 stage. Both of these unigenes are involved in important reactions in the formation of long-chain unsaturated fatty acids.

\section{Identification and characterization of genes involved in fatty acid degradation}

A total of 168 unigenes were annotated to fatty acid degradation pathways in the transcriptome database analysed in the present study, and 14 DEGs were screened and found to be significantly upregulated in fruit samples according to KEGG enrichment analysis (Additional file 14:Table S6, Fig. 2d). The expression values of these candidate DEGs in the five major organs and their TAIR10 annotations are also listed in (Additional file 15: Table S7) and (Additional file 16: Table S8). Interestingly, the major pathway of fatty acid degradation was beta-oxidation catabolism, and the main enzymes involved in each step were identified in our DEG profiles, which included long-chain acyl-CoA synthase (LACS), acyl-CoA oxidase $(A C X)$, multifunctional protein 2 (MFP2), hydroxyacyl-CoA dehydrogenase (HADH), ketoacyl-CoA thiolase (KAT) and acetoacetyl-CoA thiolase 2 (ACAT2) (Fig. 5a).

The two LACS genes were investigated in the present study, whose encoded proteins catalyse the initial reactions of the fatty acid degradation process. Cluster-12,235.42753, annotated to ZaLACS1, was only very significantly upregulated in the Fr4 stage (Fig. 5b), whereas Cluster-12, 235.42753 (ZaLACS6) was not only upregulated in the Fr4 stage but was also highly expressed in the Fr6 stage. Similar results were obtained for ZaACX3 (Cluster-12,235.39988) and ZaMFP2 (Cluster-12,235.50349), which catalyse the first and second reactions of fatty acid $\beta$-oxidation, respectively, and presented two peaks of upregulation ( $\mathrm{Fr} 4$ and Fr6). Additionally, their downstream oxidase, ZaKATII (Cluster-12,235.55699), which generates and releases CoA, was distinctively upregulated in the Fr4 and Fr6 stages. 
(a)

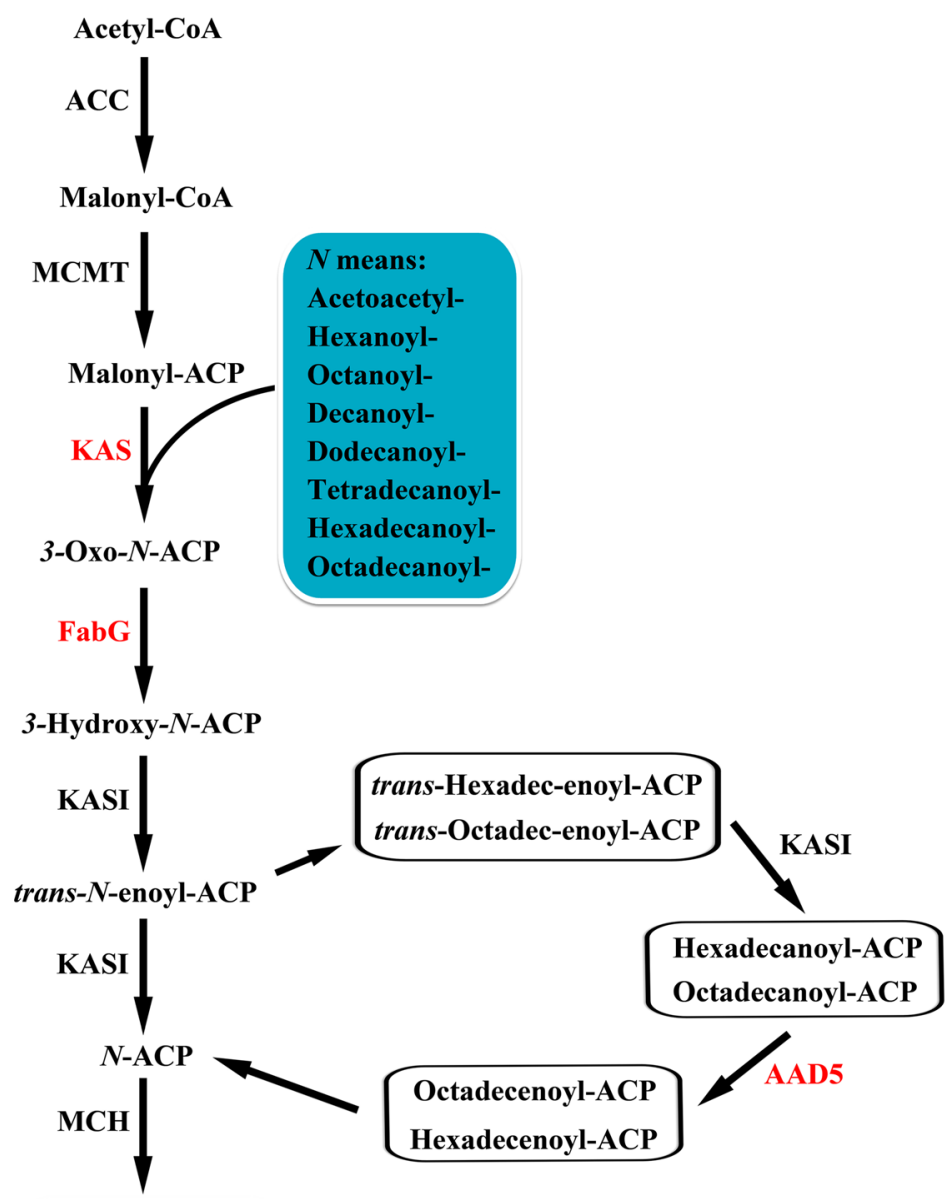

\section{Octanoic acid}

Decanoic acid

Dodecanoic acid

Tetradecanoic acid

Hexadecanoic acid

Hexadecenoic acid

Octadecanoic acid

Octadecenoic acid (b)
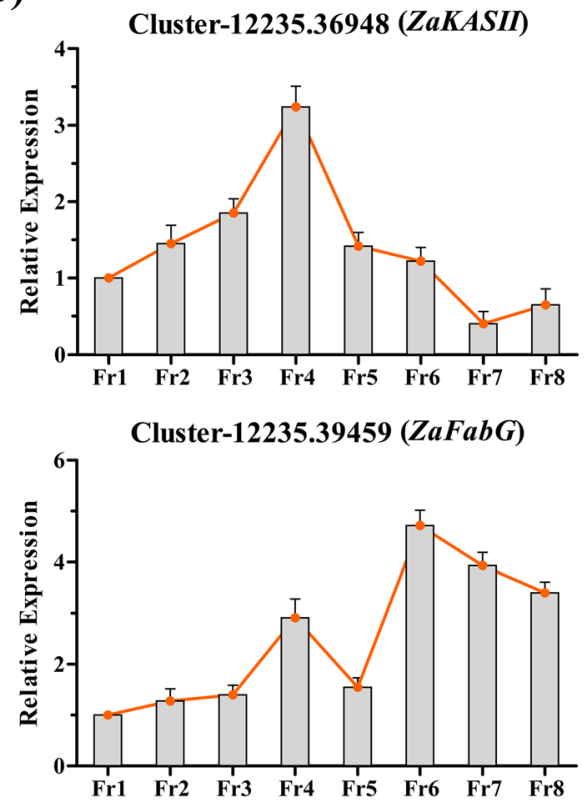

Cluster-12235.43479 (ZaAAD5)

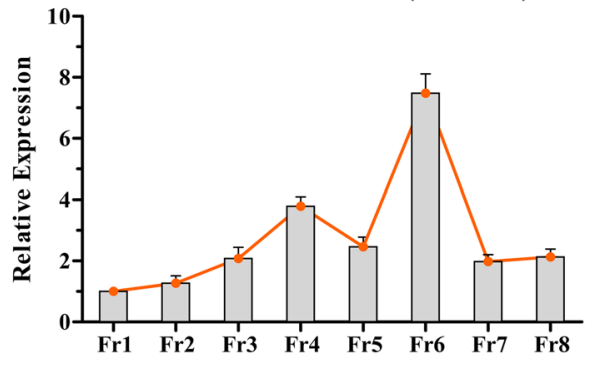

Cluster-12235.42753 (ZaAAD5)

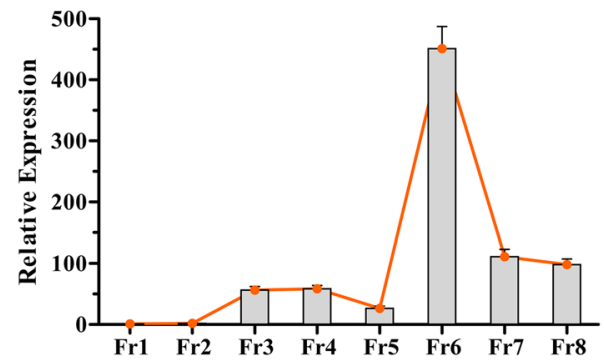

Fig. 4 The identification of genes in the pathway of fatty acid biosynthesis based on the transcriptome of $Z$. armatum. a the regulatory cascade of fatty acid biosynthesis pathway. Red fonts indicates the homologous differential expressed genes significantly up-regulated in this study and they were abbreviated as follows and they were abbreviated as follows: ACP, acyl carrier protein; KASII, Ketoacyl-ACP Synthase Il; FabG, Oxoacyl-acyl-carrier Protein Reductase; AAD5, ACP Desaturase 5. $\mathbf{b}$ the relative expression of the DEGs during fruit development and maturation process. The Fr1 was used as the control sample

Additionally, ZaACAT2 (Cluster-12,235.42348), which is involved in a key step in the transformation of acetoacetylCoA into acetyl-CoA, was still highly expressed in the Fr4 and Fr6 stages of Z. armatum.

\section{qRT-PCR validation}

To experimentally confirm the RNA-seq data, eight DEGs (with 21 terms) detected in Frvs. Ro, Frvs. St,
Frvs.LB, Frvs.ML, LBvs. Ro, MLvs.LB, MLvs. Ro, and MLvs. St were tested by qRT-PCR (Additional file 18: Table S9). These genes were randomly selected based on their high fold changes indicating their crucial functions identified in this study. The results for all of these genes were consistent and showed the same trend of up- or downregulation between the qRT-PCR and the RNA-seq platform (Additional file 19: Figure S11). The correlation 

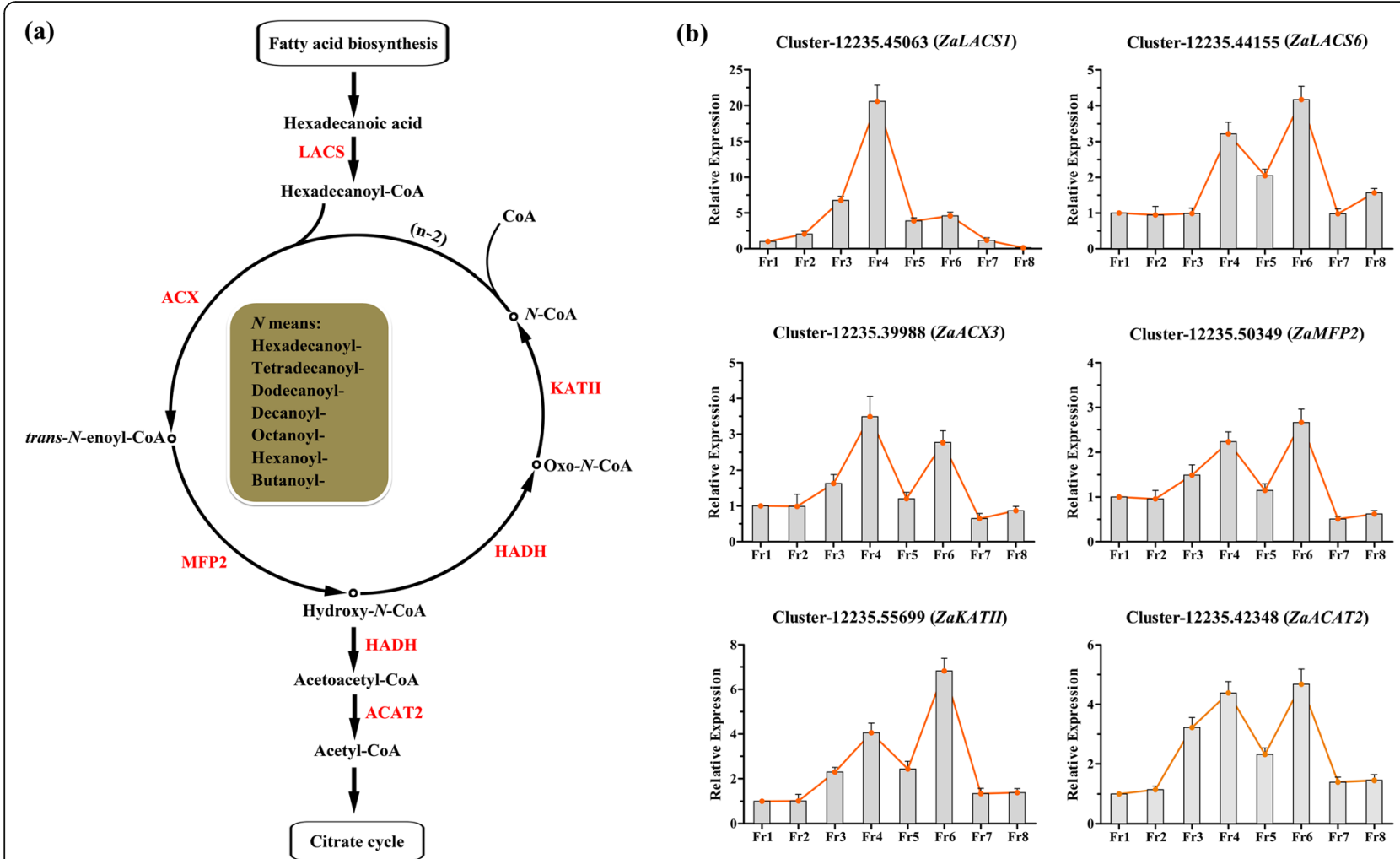

Fig. 5 The identification of genes in the pathway of fatty acid degradation based on the transcriptome of Z. armatum. a the regulatory cascade of fatty acid degradation pathway. Red fonts indicates the homologous differential expressed genes significantly up-regulated in this study and they were abbreviated as follows: LACS, Long-chain Acyl-CoA Synthetase; ACX3, Acyl-CoA Oxidase 3; MFP2, Multi-functional Protein 2; HADH, Hydroxyacyl-CoA Dehydrogenase; KATII, Ketoacyl-CoA Thiolase II; ACAT2, Acetoacetyl-CoA Thiolase 2. $\mathbf{b}$ the relative expression of the DEGs during fruit development and maturation process. The Fr1 was used as the control sample

coefficient for the two expression measurements was 0.8059 between these 24 terms $\left(R^{2}=0.8059\right)$. In summary, the results suggested that our transcriptome data accurately reflected the expression patterns of most genes in $Z$. armatum.

\section{Discussion}

In the present study, the five major organs of Z. armatum were collected to construct a comprehensive transcriptome dataset with abundant genetic information using the Illumina $\mathrm{HiSeq}^{\mathrm{Tm}} 4000$ platform in Z. armatum. All the unigenes were subjected to BLASTx searches to characterize their gene functions against five public databases, and the species distribution of the NR BLASTx matches showed that $Z$. armatum was presents a close relationship to Citrus species. In fact, both Citrus and Zanthoxylum belong to Rutaceae [25]. The consistent results indicated that Zanthoxylum is a relatively recent species that is predicted to have diverged from Citrus 36.5-37.7 million years ago [1, 26].

Additionally, the present study showed that at least 912 repeats were detectable for monomer nucleotide SSRs, whereas the di- to hexanucleotide SSRs were explored mostly in the context of 5 to 8 repeats. These results indicated that more repeats were likely to be detected for repeat types with fewer nucleotides, which is consistent with reported studies in various plants [27], and the types of SSRs are affected by the selective pressure on different genes [28]. In summary, a large number of molecular markers were detected in the present study, indicating that high variation might exist in $Z$. armatum. In a previous study, 11 pairs of primers were used to clearly distinguish Zanthoxylum bungeanum (red Sichuan pepper) from Zanthoxylum armatum (green Sichuan pepper), even differentiating various cultivars [1]. The molecular markers screened in the present study provide rich information and could contribute to the improvement of a molecular marker database that can be used for revealing high polymorphism and multiple alleles and for markerassisted selection breeding in Zanthoxylum species.

Additionally, this study identified a large number of organ-specific unigenes that were significantly associated with development and other biological processes. All the KEGG enrichments for each organ were consistent with previous studies showing that components responsible for the special numbing taste, including volatile oils, 
alkaloids, terpenoids, acid amide phenol components and so on [6,7], accumulate at high levels in the fruits, leaves and roots of $Z$. armatum [8]. These results could also reveal many candidates and underlying specific promoters related to the special numbing taste, and further research could focus on confirming their molecular functions in model plants (such as Arabidopsis thaliana) and $Z$. armatum to provide more genetic information about these genes.

The fruit of $Z$. armatum is the main organ in which numbing taste-related compounds accumulate [8]. Thus, the present study screened DEGs related to terpenoid biosynthesis and fatty acid biosynthesis in the comparisons of fruit samples with other organs (Fr vs. Ro, Fr vs. St, Fr vs. LB, and Fr vs. ML). This approach identified 53 DEGs related to 12 enzymes involved in the terpenoid biosynthesis process, covering almost all of the important enzymes associated with terpenoid backbone biosynthesis. These results indicated that the fruit is a key location of the synthesis of terpenoid compounds in $Z$. armatum. Therefore, the eight samples were harvested to further identify their expression patterns in fruit development and maturation stages. Eight important candidate genes were selected for qRT-PCR analysis, including ZaDXS, ZaISPE, ZaGCPE, ZaMVK1, ZaPMVK, ZaTPSO3 and ZaGGPPS12. Two unigenes, Cluster-12,235.38535 and Cluster-12,235.46034, were annotated to deoxy- $D$-xylulose phosphate synthase $(D X S)$, which is recognized as a rate-limiting enzyme that catalyses the first step in the MEP pathway [29]. The functions of DXS have been characterized in multiple plants. The overexpression of LiDXS from Lilium 'Siberia' substantially increases the diterpene content of tobacco [29]. Other studies have also reported that DXS plays a crucial role in the biosynthesis of the monoterpene precursor [30-33]. Additionally, GbMVK is highly expressed in the leaves, roots and stems of Ginkgo biloba, which is related to the production of the main active substance in terpene trilactone biosynthesis [34]. In the classical MVA pathway, PMVK can catalyse the transformation of mevalonate- $5 P$ into mevalonate-5PP, which is subsequently subjected to decarboxylation catalysed by mevalonate 5-diphosphate decarboxylase [14]. Recently, Henry et al. found that PMVK is a key regulatory hub in controlling the flux through the plant MVA pathway [14]. Moreover, Cluster-12,235.40340 was annotated to geranyl-geranyl diphosphate synthase 12 (GGPPS12). GGPPS is composed of an inactive small subunit and an active large subunit and generates important substrates associated with the biosynthesis of monoterpene compounds in various plants [35-37]. ZaTPSO3 (Cluster$12,235.42010)$ was also identified in this study, which related to a typical monoterpene synthase that catalyses the transformation GPP into limonene as well as other related compounds [22]. Previous studies have shown that TPS family members are the main contributors to the production of monoterpenes and sesquiterpenes in the plant kingdom [36]. In the present study, it was notable that all eight of these genes were significantly upregulated in the Fr4 stage but downregulated in the preceding and subsequent stages of fruit development and maturation. A consistent result was that terpenoids predominantly accumulated in the mid-developmental period of the fruit maturation process in Zanthoxylum bungeanum [22]. Similar expression patterns can be detected during fruit development in finger citron [38], tomato [39] and other species [40]. Considering the results of previous work and the present study together, we suggest that forty days after fertilization could be a core period for the initiation of the biosynthesis of terpenoid compounds and their accumulation in the fruit development and maturation process of $Z$. armatum. In further studies, a strategic approach will be to measure the concentrations of various terpenoid compounds in fruit differentiation stages via metabolomics analysis coupled with high-performance liquid chromatography (HPLC) fingerprinting $[5,41]$, and the functions of crucial genes could also be evaluated with transgenic methods to verify the present results in depth.

Finally, this study also screened 34 DEGs associated with fatty acid biosynthesis, elongation and degradation pathways from the 347 background unigenes in the present transcriptome dataset (Additional file 14: Table S6). According to the RNA-seq analysis, all of these DEGs were exclusively upregulated in fruit samples. Similarly, ten candidate genes were investigated for their expression patterns in fruit development and maturation stages. The function of KASII has been fully elucidatedin many different higher plants, and its main role is to catalyse the elongation of palmitoyl-ACP [42]. Four unigenes were annotated to FabG in the KEGG pathways, which can reduce 3-Oxo- $N$-ACP to form 3-hydroxy- $N$-ACP [43]. However, the genetic information and gene names of these unigenes were not annotated in the TAIR10 database. The family members of $A A D 5$ are responsible for long-chain unsaturated fatty acid biosynthesis and are predominantly expressed throughout seed developmental stages. The aad5 mutation of Arabidopsis can cause a significant reduction in the concentration of C18:1 fatty acids [44]. The present study showed that ZaKASII was significantly upregulated in the Fr4 stage, whereas both ZaAAD5 genes were exclusively upregulated in the Fr6 stage. Additionally, ZaFabG showed two expression peaks, not only being upregulated in the Fr4 stage but also showing high expression in the Fr6-Fr8 stages. DEGs related to the fatty acid degradation pathway were also identified in fruit development and maturation stages, including ZaLACS, ZaACX3, ZaKATII and ZaACAT2. Multiple studies have explored the genetic functions of these genes involved in fatty acid accumulation and degradation 
in Arabidopsis thaliana and other plants [45-48]. Additionally, ACAT2 is typically related to terpenoid biosynthesis in the mevalonate pathway, where it can reversibly catalyse formation of acetyl-CoA from acetoacetyl-CoA [12]. It is worth noting that almost all of these genes exhibited two upregulation peaks in the Fr4 and Fr6 stages, except for ZaLACS1, which was only very significantly upregulated in the Fr4 stage. These results were consistent with the expression patterns of the genes in fatty acid biosynthesis pathways detected in this study (Figs. 4 and 5). In a previous study, genes related to fatty acid biosynthesis were grouped into two categories and detected in the early or later stages of seed development in tree peony [49]. Similarly, the coordinated regulation of multiple genes has been shown to promote the high accumulation of longchain unsaturated fatty acids in the seeds of tea oil camellia [50], and consistent results have been obtained in Brassica napus [51, 52], Glycine max [53], and Linum usitatissimum [54]. Above all, the present results indicated that 40 days after fertilization could be a key point at which the first few steps of fatty acid biosynthesis are promoted in Z. armatum, and the subsequent reactions could be catalysed and completed in the stage occurring 62 days after fertilization, especially for long-chain fatty acid biosynthesis.

The present study only investigated the expression patterns of certain crucial candidate genes involved in terpenoid compound and fatty acid biosynthesis and metabolism. Further study could focus on identifying the relative expression levels of more genes related to the pathways of alkaloids, coumarins, acid amide phenol components and so on from the present transcriptome dataset. Additionally, a further study could be devoted to confirming the molecular functions of these genes via the overexpression of the genes driven by the $35 \mathrm{~S}$ promoter and/or their silencing by hairpin RNAs in transgenic $Z$. armatum plants, which might reveal more genetic information underlying the regulatory mechanism associated with the special aromatic taste of $Z$. armatum.

\section{Conclusions}

The present study achieved de novo transcriptome assembly for five major organs in Z. armatum. A total of 111, 318 nonredundant unigenes were generated, with an average length of $1014 \mathrm{bp}, 73,426$ of which could be functionally annotated in at least one database. Additionally, a large number of SSRs were obtained to improve our understanding of the phylogeny and genetics of some important traits of $Z$. armatum. The organ-specific unigenes of the five major samples were screened and annotated via GO and KEGG enrichment analysis. Additionally, 1876 unigenes were found to be exclusively up- or downregulated in fruit samples, 53 of which were identified as candidate unigenes for 12 enzymes involved in terpenoid biosynthesis, 34 of which were significantly annotated to fatty acid biosynthesis, elongation and degradation pathways. Furthermore, qRT-PCR detection was conducted in eight fruit development and maturation stages. Forty days after fertilization could be a crucial period for the initiation of the biosynthesis of terpenoid compounds and their accumulation in the fruit development and maturation process of $Z$. armatum. However, the Fr4 stage could be a key point at which the upstream steps of fatty acid biosynthesis are promoted in $Z$. armatum, and the subsequent reactions could be significantly activated in the Fr6 stage, especially long-chain unsaturated fatty acid biosynthesis.

The present dataset provides a reference transcriptome for the genomic database of $Z$. armatum for future studies. To the best of our knowledge, this study is the first to perform a comprehensive analysis revealing the underlying genes related to the special numbing taste in $Z$. armatum. The assembled transcriptome profiles expand the available genetic information for $Z$. armatum and will contribute to gene functional studies, which will aid in the engineering of high-quality varieties of $Z$. armatum.

\section{Methods \\ Plant material collection}

Three-year-old $Z$. armatum planted at a forestry trial base of Sichuan Agricultural University $\left(30.60^{\circ} \mathrm{N}, 103.65^{\circ} \mathrm{E}\right)$ in Chengdu City, Sichuan Province, China, was selected as the experimental material in the present study. The material has been authenticated by the Forestry Variety Certification Committee of Sichuan Province, and the deposition number is SV-ZA-002-2018. This improved variety, $Z$. armatum 'Hanyuan putaoqing', was bred by our coauthor Professor Wei Gong (from Sichuan Agricultural University). The trial site is located in a subtropical humid monsoon climate area, with an annual average sunshine of $>1160 \mathrm{~h}$, mean annual rainfall of $1012 \mathrm{~mm}$, and a mean annual temperature of $15.9^{\circ} \mathrm{C}$. Five organs, including the roots (Ro), stems (St), leaf buds (LB), mature leaves (ML) and fruits (Fr), were harvested for RNA-seq analysis in May 2019. These samples were collected at approximately 60 days after fertilization, when the fruits had reached full size and were rapidly accumulating aromatic compounds and fatty acids. Additionally, the fruit samples were harvested approximately every 10 days during fruit development and maturation from April to July (Additional file 17: Figure S10). In total, eight samples (Fr1 to Fr8) were obtained to investigate the genes associated with terpenoid compound and fatty acid metabolism in the present study. Each sample consisted of material collected from three individual plants, which was mixed together as a biological replicate, and three independent biological replicates were performed. All samples were flash frozen in liquid nitrogen and stored at $-80^{\circ} \mathrm{C}$ until being used for RNA extraction. 
Total RNA extraction and transcriptome sequencing analysis The total RNA of each sample was extracted separately using the Magen Plant RNA Kit (HiPure HP Plant RNA Mini Kit, Guangzhou) following the instructions of the manufacturer. The quality of the total RNA was investigated by using agarose gel electrophoresis and a Nanodrop 2100 spectrophotometer (Agilent, USA). The 15 cDNA libraries in this study (Ro_1, Ro_2, Ro_3, St_1, St_2, St_3, LB_1, LB_2, LB_3, ML_1, ML_2, ML_3, Fr_1, Fr_2, and Fr_3) were constructed and sequenced using the Illumina $\mathrm{HiSeq}^{\mathrm{Tm}} 4000$ (Illumina, USA) platform at Novegene Bioinformatics Technology Co. Ltd. (Beijing, China). Sequence adaptors, reads with more than $10 \% \mathrm{~N}$ bases and low-quality reads ( $Q$ phred $\leq 20$ for $>50 \%$ read) were removed to obtain clean data according to the method described by Lv et al. [55]. After quality control procedures, the unigenes were de novo assembled using Trinity software [56]. All of the transcriptome data were used as a reference dataset to calculate the read count of each unigene among the samples and converted to the expected number of fragments per kilobase of transcript sequence per million base pairs sequenced (FPKM) as described by Trapnell et al. [57].

\section{Gene functional annotation and DEG analysis}

To obtain comprehensive functional information, all unigenes were aligned by BLASTx to the NCBI nonredundant protein sequence (NR), NCBI nucleotide sequence (NT), Protein family (Pfam), KEGG Ortholog (KO) and euKaryotic Ortholog Groups (KOG) databases with a threshold E-value of $10^{-5}$. Additionally, the functional annotation and classification of all the unigenes were also performed according to the Gene Ontology database (GO, http://www.geneontology.org/) and the Kyoto Encyclopedia of Genes and Genomes database (KEGG, https://www.kegg.jp/).

The differential expression analysis of the samples with three biological replications was conducted using edgeR [58]. The fold change (FC) is the gene expression difference between different samples. The organ-specific unigenes of the five major samples were screened according to a $q$ value $<0.05$ and $\mid \log 2$ (fold change) $\mid>5$, and the differentially expressed genes (DEGs) between different samples were identified according to a $q$ value $<0.05$ and $\mid \log 2$ (fold change) $\mid>1$. All of the up- or downregulated genes described in the present study are illustrated by the first comparison component. GOSeq and KOBAS software were used to estimate the statistical enrichment of DEGs in GO terms and KEGG pathways, respectively $[59,60]$. A corrected $p$-value of 0.05 was set as the threshold for the significant enrichment of GO terms and KEGG pathways. BLASTx alignments were performed for the DEGs in the TAIR 10 database (https:// www.arabidopsis.org/). The volcano plot and heat maps of the DEGs identified in this study were drawn with $\mathrm{R}$ software (R-2.15.3-win) according to the procedure described by Silva et al. [61].

\section{Identification of simple sequence repeats}

The Perl script MISA was used to identify the simple sequence repeats (SSRs) according to the procedure described by Wang et al. [62]. Similar criteria to those employed in previous studies were used for the screening of high-quality SSRs in the five different samples collected in this study [20, 27].

\section{Quantitative real-time PCR analysis}

To validate the transcriptome results, a total of eight DEGs (21 terms) detected in Frvs. Ro, Frvs. St, Frvs.LB, Frvs.ML, LBvs. Ro, MLvs.LB, MLvs. Ro, and MLvs. St were selected for quantitative real-time PCR (qRT-PCR) analysis using the same plant materials employed for RNA sequencing. These genes were randomly selected based on their high fold changes and crucial functions identified in this study (Additional file 18: Table S9). Three biological replicates were carried out for the different organ samples, with three technological replications for each gene. Moreover, the DEGs involved in terpenoid compound and fatty acid metabolism were selected for qRT-PCR analysis using eight samples related to fruit development and maturation. cDNA synthesis was performed using the PrimeScript ${ }^{\circledR}$ II First Strand cDNA Synthesis Kit (TaKaRa, Japan). The specific primers were designed with Primer Premier 5.0, and the amplified PCR products varied from 80 to $300 \mathrm{bp}$ in length (Additional file 20: Table S10). qRT-PCR was performed in a Bio-Rad CFX96 ${ }^{\mathrm{Tm}}$ system (Bio-Rad, America) with SYBR Premix Ex $\mathrm{Taq}^{\mathrm{TM}}$ II (TaKaRa, Japan). ZaUBQ was selected as an internal control according to a previous study [2]. The $2^{-\Delta \Delta C t}$ method was used to calculate the relative expression levels of the genes in different samples [63]. Fr1 (Fruit 1) was used as a control sample to estimate the relative expression of the DEGs associated with terpenoid compounds and fatty acid metabolism during the fruit development and maturation process in this study. GraphPad Prism 5 was used to draw the figures [64].

\section{Supplementary information}

Supplementary information accompanies this paper at https://doi.org/10. 1186/s12864-020-6521-4

Additional file 1: Table S1. The quality of RNA extration.

Additional file 2: Table S2. Overview of the sequencing data of Zanthoxylum armatum transcriptome.

Additional file 3: Figure S1. The length distribution of transcripts (a) and unigenes (b). The inserts show the frequency distribution of the transcript length (a) and of the unigene length (b).

Additional file 4: Table S3. The summary of SSR in the total of unigenes. Additional file 5: Figure S2. The overview of Zanthoxylum armatum transcriptome assembly and the characteristics of the homology search 
of unigenes. (a) e-value distributions of the best BLAST hits for each unigene against the NR database. (b) similarity distribution of the best BLAST hits for each unigene against the NR database. (c) venn diagram showing the BLAST searches of the Zanthoxylum armatum transcriptome against the five public databases. (d) species distribution of the best BLAST hit for each unigene against the NR database.

Additional file 6: Figure S3. Gene ontology distributions for the transcriptome of five major samples in Z. armatum. Main functional categories of the transcriptome related to plant physiology of the biological process, cellular component, and molecular function. The abscissas show the number of unigenes, and one unigene may be associated with different $\mathrm{GO}$ terms.

Additional file 7 Figure S4. The KEGG pathway for the transcriptome of five major samples in $Z$. armatum. The unigenes were divided into five clusters according to KEGG metabolism pathways, A: Cellular processes, B: Environmental information processing, C: Genetic information processing, D: Metabolism, E: Organismal systems.

Additional file 8: Table S4. KEGG annotation of nonredundant unigenes in Zanthoxylum armatum.

Additional file 9: Figure S5. Heat map representation and hierarchical clustering of putative genes involved in fatty acid metabolism pathways.

Additional file 10: Figure S6. Heat map representation and hierarchical clustering of putative genes involved in terpenoid compounds biosynthesis pathways.

Additional file 11: Figure S7. The pearson correlation coefficient $\left(R^{2}\right)$ was used to estimate the difference between the replicates of each tissue. The number between these two samples is given in the plot. The color represents $\mathrm{R}$ value, which shows high correlation in blue between two samples, while low correlation in white.

Additional file 12: Figure S8. The volcano plot of differential expressed genes (DEGs) associated with organ-specific unigenes. (a)-(e) represent the organ-specific unigenes in Ro, St, LB, ML and Fr, respectively. The abscissa shown the fold change of DEGs in each comparison and the ordinate shows the significance of DEGs, $a<0.05$. The red dot was up regulation, the blue dot was down regulation, and the green dot was not significant difference. $(f)$ was the percentage of unigenes expressed in each organ.

Additional file 13: Table S5. The significant pathways of KEGG enrichment related to organ-specific unigenes in Zanthoxylum armatum.

Additional file 14: Table S6. The significant paythways of KEGG enrichment related to the unigenes only up- or down- regulation in fruit samples of Zanthoxylum armatum.

Additional file 15: Table S7. The FPKM of the unigenes detected in the significant KEGG enrichment pathways of only up-regulation in fruit samples of Zanthoxylum armatum.

Additional file 16: Table S8. The TAIR annotation of the DEGs associated with terpenoid compounds and fatty acids biosynthesis and metabolism in fruit samples of Zanthoxylum armatum.

Additional file 17: Figure S10. The samples involved in fruit development and maturation in Z. armatum. (a)-(h) represent the samples collected from Fr1 to Fr8, respectively. All the fruit samples were harvested from April to July 2019. The bar is $2 \mathrm{~mm}$.

Additional file 18: Table S9. The RNA-seq data of the DEGs selected for qRT-PCR validation.

Additional file 19: Figure S11. The correlation between $q R T-P C R$ and RNA-seq data. Correlation between qRT-PCR and RNA-seq data of eight DEGs (21 terms) selected in Frvs. Ro, Frvs. St, Frvs.LB, Frvs.ML, LBvs. Ro, MLvs.LB, MLvs. Ro, and MLvs. St, including 6 down-regulation and 15 upregulation. Pearson correlation coefficient $=0.8059(p<0.05)$.

Additional file 20: Table S10. The primers of qRT-PCR used for validation of expression trend in Zanthoxylum armatum.

\section{Abbreviations}

AAD5: ACP desaturase 5: ACAT2: Acetoacetyl-CoA thiolase 2; ACP: acyl carrier protein; ACX3: Acyl-CoA oxidase 3; DEG: Differential expressed genes; DXS: Deoxy-D-xylulose 5-phosphate synthase; FabG: Oxoacyl-acyl-carrier protein reductase; FC: Fold change; GCPE: Hydroxy-methylbut-enyl diphosphate synthase; GGPPS12: Geranylgeranyl diphosphate synthase; GO: Gene ontology; HADH: Hydroxyacyl-CoA dehydrogenase; HDR: Hydroxymethylbut-enyl diphosphate reductase; HMGR: Hydroxy-methyl-glutaryl-CoA reductase; HMGS: Hydroxy-methyl-glutaryl-CoA synthase;

ISPE: Diphosphocytidyl-methyl-D-erythritol kinase; ISPF: Isoprenoid F; KASII: Ketoacyl-ACP synthase II; KATII: Ketoacyl-CoA thiolase II; KEGG: Kyoto encyclopedia of genes and genomes; LACS: Long-chain acyl-CoA synthetase; MFP2: Multi-functional protein 2; MVK1: Mevalonate kinase 1; NCBI: National center for biotechnology information; PMVK: Phosphomevalonate Kinase; SNP: Single nucleotide polymorphism; SSR: Simple sequence repeats; TAIR: The Arabidopsis information resource; Z. armatum: Zanthoxylum armatum

\section{Acknowledgments}

We appreciate Prof. Guojiang Wu (From Chinese Academy of Sciences) for useful discussions, and Nature Research Editing Service (https:// authorservices.springernature.com) for its linguistic assistance during the preparation of this manuscript.

\section{Authors' contributions}

$\mathrm{W}-\mathrm{KH}$ and $\mathrm{A}-\mathrm{MW}$ formulated and designed the experiments; $\mathrm{W}-\mathrm{KH}$ and $\mathrm{H}-\mathrm{YL}$ collected the materials; $\mathrm{W}-\mathrm{KH}, \mathrm{J}-\mathrm{WL}, \mathrm{J}-\mathrm{XZ}$, and L-ZZ performed the experiments; W-KH, F-YZ, J-YW, YZ and Q-MQ draw the figures and analyzed the data; W-KH wrote the paper; WG and X-YC revised and proofread the paper. All authors read and approved the final manuscript.

\section{Funding}

This work was supported by the National Key Research and Development Program of China (WG, 2018YFD1000605), and the Forest and Bamboo Breeding Project of Sichuan Province for the Fifth Year Plan (WG 2016NYZ0035). The funding bodies had no role in the experimental design, sample collection, data analysis and interpretation, and manuscript writing.

\section{Availability of data and materials}

The data supporting the results presented in this article are included as additional files. All RNA-seq data in this study are archived in NCBI GEO database with the accession number GSE142491

\section{Ethics approval and consent to participate}

Not applicable.

Consent for publication

Not applicable.

\section{Competing interests}

The authors declare that they have no competing interest.

\section{Author details}

${ }^{1}$ Key Laboratory of Ecological Forestry Engineering of Sichuan Province, College of Forestry, Sichuan Agricultural University, Chengdu 611130, China. ${ }^{2}$ Guangdong Key Laboratory for Innovative Development and Utilization of Forest Plant Germplasm, State Key Laboratory for Conservation and Utilization of Subtropical Agro-Bioresources, College of Forestry and Landscape Architecture, South China Agricultural University, Guangzhou 510642, China. ${ }^{3}$ Agricultural Technology Extension Center in Yantan District, Zigong 643030, China.

Received: 14 November 2019 Accepted: 20 January 2020

Published online: 28 January 2020

\section{References}

1. Feng S, Zhao L, Liu Z, Liu Y, Yang T, Wei A. De novo transcriptome assembly of Zanthoxylum bungeanum using Illumina sequencing for evolutionary analysis and simple sequence repeat marker development. Sci Rep UK. 2017:7:16754.

2. Fei X, Shi Q, Yang T, Fei Z, Wei A. Expression stabilities of ten candidate reference genes for RT-qPCR in Zanthoxylum bungeanum maxim. Molecules. 2018;23(4):802

3. Phuyal N, Jha PK, Prasad Raturi P, Rajbhandary S. Zanthoxylum armatum DC: current knowledge, gaps and opportunities in Nepal. J Ethnopharmacol. 2019;229:326-41. 
4. Nooreen Z, Tandon S, Yadav NP, Kumar P, Xuan TD, Ahmad A. Zanthoxylum: a review of its traditional uses, naturally occurring constituents and pharmacological properties. Curr Org Chem. 2019;23(12): 1307-41.

5. Ke J, Qu Y, Li S, Shen G, Chen A, Luo Q, Liu X, Wu H, Li M, Pu B, Ye M, Zhang Z. Application of HPLC fingerprint based on acid amide components in Chinese prickly ash (Zanthoxylum). Ind Crop Prod. 2018;119:267-76.

6. Bader M, Stark TD, Dawid C, Loesch S, Hofmann T. All-trans-configuration in Zanthoxylum alkylamides swaps the tingling with a numbing sensation and diminishes salivation. J Agr Food Chem. 2014;62(12):2479-88.

7. Yang X. Aroma constituents and alkylamides of red and green huajiao (Zanthoxylum bungeanum and Zanthoxylum schinifolium). J Agr Food Chem. 2008;56(5):1689-96.

8. Z Zhang M, Wang J, Zhu L, Li T, Jiang W, Zhou J, Peng W, Wu C. Zanthoxylum bungeanum Maxim. (Rutaceae): a systematic review of its traditional uses, botany, phytochemistry, pharmacology, pharmacokinetics, and toxicology. Int J Mol Sci. 2017:18(10):2172.

9. Yang L, Li R, Tan J, Jiang Z. Polyphenolics composition of the leaves of Zanthoxylum bungeanum maxim. Grown in Hebei, China, and their radical scavenging activities. J Agr Food Chem. 2013;61(8):1772-8.

10. Alam F, Sagib QNU, Ashraf M. Zanthoxylum armatum DC extracts from fruit, bark and leaf induce hypolipidemic and hypoglycemic effects in micein vivo and in vitro study. BMC Complem Altern M. 2018;18:68.

11. Godshaw J, Hjelmeland AK, Zweigenbaum J, Ebeler SE. Changes in glycosylation patterns of monoterpenes during grape berry maturation in six cultivars of Vitis vinifera. Food Chem. 2019;297:124921.

12. Si Y, Wen H, Li Y, He F, Li J, Li S, He H. Liver transcriptome analysis reveals extensive transcriptional plasticity during acclimation to low salinity in cynoglossus semilaevis. BMC Genomics. 2018;19:464.

13. Pankratov I, McQuinn R, Schwartz J, Bar E, Fei Z, Lewinsohn E, Zamir D, Giovannoni JJ, Hirschberg J. Fruit carotenoid-deficient mutants in tomato reveal a function of the plastidial isopentenyl diphosphate isomerase (IDI1) in carotenoid biosynthesis. Plant J. 2016;88(1):82-94.

14. Henry LK, Thomas ST, Widhalm JR, Lynch JH, Davis TC, Kessler SA, Bohlmann J, Noel JP, Dudareva N. Contribution of isopentenyl phosphate to plant terpenoid metabolism. Nat Plants. 2018:4(9):721-9.

15. Xia L, You J, Li G, Sun Z, Suo Y. Compositional and antioxidant activity analysis of Zanthoxylum bungeanum seed oil obtained by supercritical $\mathrm{CO}_{2}$ fluid extraction. J Am Oil Chem Soc. 2011;88:23-32.

16. Huo LX, Liu YL, Wei AZ. Geographical variations in the fatty acids of Zanthoxylum seed oils: a chemometric classification based on the random forest algorithm. Ind Crop Prod. 2019;134:146-53.

17. Wu J, Huang J, Hong Y, Zhang H, Ding M, Lou H, Hu Y, Yu W, Song L. De novo transcriptome sequencing of torreya grandis reveals gene regulation in sciadonic acid biosynthesis pathway. Ind Crop Prod. 2018;120:47-60.

18. Dussert S, Guerin C, Andersson M, Joet T, Tranbarger TJ, Pizot M, Sarah G, Omore A, Durand-Gasselin T, Morcillo F. Comparative transcriptome analysis of three oil palm fruit and seed tissues that differ in oil content and fatty acid composition. Plant Physiol. 2013;162(3):1337-58.

19. Xiao Y, Xia W, Mason AS, Cao Z, Fan H, Zhang B, Zhang J, Ma Z, Peng M, Huang D. Genetic control of fatty acid composition in coconut (Cocos nucifera), african oil palm (Elaeis guineensis), and date palm (Phoenix dactylifera). Planta. 2019;249(2):333-50

20. Hu X, Pan B, Fu Q, Niu L, Chen M, Xu Z. De novo transcriptome assembly of the eight major organs of sacha inchi (Plukenetia volubilis) and the identification of genes involved in a-linolenic acid metabolism. BMC Genomics. 2018;19:380.

21. Wang J, Liu J, Chen K, Li H, He J, Guan B, He L. Comparative transcriptome and proteome profiling of two Citrus sinensis cultivars during fruit development and ripening. BMC Genomics. 2017;18:984.

22. Shi J, Fei $X$, Hu Y, Liu Y, Wei A. Identification of key genes in the synthesis pathway of volatile terpenoids in fruit of Zanthoxylum bungeanum maxim. Forests. 2019;10(4):328.

23. Zhang $L$, Lin Q, Feng $Y$, Fan $X$, Zou F, Yuan D, Zeng $X$, Cao $H$. Transcriptomic identification and expression of starch and sucrose metabolism genes in the seeds of Chinese chestnut (Castanea mollissima). J Agr Food Chem. 2015;63(3):929-42.

24. Cao M, Zhang S, Li M, Liu Y, Dong P, Li S, Kuang M, Li R, Zhou Y. Discovery of four novel viruses associated with flower yellowing disease of green Sichuan pepper (Zanthoxylum armatum) by virome analysis. Viruses. 2019; 11(8):696.
25. Appelhans MS, Reichelt N, Groppo M, Paetzold C, Wen J. Phylogeny and biogeography of the pantropical genus Zanthoxylum and its closest relatives in the proto-Rutaceae group (Rutaceae). Mol Phylogenet Evol. 2018;126:31-44.

26. Tian J, Feng S, Liu Y, Zhao L, Tian L, Hu Y, Yang T, Wei A. Single-molecule long-read sequencing 696 of Zanthoxylum bungeanum Maxim. transcriptome: identification of aroma-related genes. Forests. 2018;9(12):765.

27. Luo $X, X u$ L, Liang D, Wang $Y$, Zhang W, Zhu X, Zhu Y, Jiang H, Tang M, Liu L. Comparative transcriptomics uncovers alternative splicing and molecular marker development in radish (Raphanus sativus L.). BMC Genomics. 2017; 18:505.

28. Bian Y, Ballington J, Raja A, Brouwer C, Reid R, Burke M, Wang X, Rowland LJ, Bassil N, Brown A. Patterns of simple sequence repeats in cultivated blueberries (Vaccinium section Cyanococcus spp.) and their use in revealing genetic diversity and population structure. Mol Breeding. 2014;34(2):675-89.

29. Zhang T, Sun M, Guo Y, Shi X, Yang Y, Chen J, Zheng T, Han Y, Bao F, Ahmad S. Overexpression of LiDXS and LiDXR from lily (Lilium 'Siberia') enhances the terpenoid content in tobacco flowers. Front Plant Sci. 2018;9:909.

30. Celedon JM, Bohlmann J. Oleoresin defenses in conifers: chemical diversity, terpene synthases and limitations of oleoresin defense under climate change. New Phytol. 2019. https://doi.org/10.1111/nph.15984.

31. Zhang E, Chai F, Zhang H, Li S, Liang Z, Fan P. Effects of sunlight exclusion on the profiles of monoterpene biosynthesis and accumulation in grape exocarp and mesocarp. Food Chem. 2017;237:379-89.

32. Yuan Y, Yu M, Jia Z, Song X, Liang Y, Zhang J. Analysis of Dendrobium huoshanense transcriptome unveils putative genes associated with active ingredients synthesis. BMC Genomics. 2018;19:978.

33. Mendoza-Poudereux I, Muñoz-Bertomeu J, Arrillaga I, Segura J. Deoxyxylulose 5-phosphate reductoisomerase is not a rate-determining enzyme for essential oil production in spike lavender. J Plant Physiol. 2014; 171(17):1564-70.

34. Chen Q, Yan J, Meng X, Xu F, Zhang W, Liao Y, Qu J. Molecular cloning, characterization, and functional analysis of acetyl-CoA C-acetyltransferase and mevalonate kinase genes involved in terpene trilactone biosynthesis from Ginkgo biloba. Molecules. 2017;22:74.

35. Wei G, Tian P, Zhang F, Qin H, Miao H, Chen Q, Hu Z, Cao L, Wang M, Gu X, Huang S, Chen M, Wang G. Integrative analyses of nontargeted volatile profiling and transcriptome data provide molecular insight into VOC diversity in cucumber plants (Cucumis sativus). Plant Physiol. 2016;172(1):603-18.

36. Rai A, Smita SS, Singh AK, Shanker K, Nagegowda DA. Heteromeric and homomeric geranyl diphosphate synthases from Catharanthus roseus and their role in monoterpene indole alkaloid biosynthesis. Mol Plant. 2013;6(5): 1531-49.

37. Kumar SR, Shilpashree HB, Nagegowda DA. Terpene moiety enhancement by overexpression of geranyl (geranyl) diphosphate synthase and geraniol synthase elevates monomeric and dimeric monoterpene indole alkaloids in transgenic Catharanthus roseus. Front Plant Sci. 2018:9:942.

38. Xu Y, Zhu C, Xu C, Sun J, Grierson D, Zhang B, Chen K. Integration of metabolite profiling and transcriptome analysis reveals genes related to volatile terpenoid metabolism in finger citron (C. medica var. Sarcodactylis). Molecules. 2019;24(14):2564.

39. Tohge T, Alseekh S, Fernie AR. On the regulation and function of secondary metabolism during fruit development and ripening. J Exp Bot. 2014;65(16): 4599-611.

40. El Hadi M, Zhang F, Wu F, Zhou C, Tao J. Advances in fruit aroma volatile research. Molecules. 2013;18(7):8200-29.

41. Plazas E, Casoti R, Avila Murillo M, Batista Da Costa F, Cuca LE. Metabolomic profiling of Zanthoxylum species: identification of anti-cholinesterase alkaloids candidates. Phytochemistry. 2019;168:112128.

42. Liu Q, Wu M, Zhang B, Shrestha P, Petrie J, Green AG, Singh SP. Genetic enhancement of palmitic acid accumulation in cotton seed oil through RNAi down-regulation of ghKAS2 encoding beta-ketoacyl-ACP synthase ॥ (KASII). Plant Biotechnol J. 2017;15(1):132-43.

43. Venkatesan R, Sah-Teli SK, Awoniyi LO, Jiang G, Prus P, Kastaniotis AJ, Hiltunen JK, Wierenga RK, Chen Z. Insights into mitochondrial fatty acid synthesis from the structure of heterotetrameric 3-ketoacyl-ACP reductase/ 3R-hydroxyacyl-CoA dehydrogenase. Nat Commun. 2014;5:4805.

44. Jin C, Li D, Gao C, Liu K, Qi S, Duan S, Li Z, Gong J, Wang J, Hai J, Chen M. Conserved function of acyl-acyl carrier protein desaturase 5 on seed oil and oleic acid biosynthesis between Arabidopsis thaliana and Brassica napus. Front Plant Sci. 2017:8:1319. 
45. Zhao L, Haslam TM, Sonntag A, Molina I, Kunst L. Functional overlap of longchain acyl-coa synthetases in Arabidopsis. Plant Cell Physiol. 2019;60(5):1041-54.

46. Khan BR, Adham AR, Zolman BK. Peroxisomal acyl-coa oxidase 4 activity differs between Arabidopsis accessions. Plant Mol Biol. 2012;8(1-2):45-58.

47. Wiszniewski AAG, Bussell JD, Long RL, Smith SM. Knockout of the two evolutionarily conserved peroxisomal 3-ketoacyl-coa thiolases in Arabidopsis recapitulates the abnormal inflorescence meristem 1 phenotype. J Exp Bot. 2014;65(22):6723-33.

48. Carrie C, Murcha MW, Millar AH, Smith SM, Whelan J. Nine 3-ketoacyl-coa thiolases (KATs) and acetoacetyl-coa thiolases (ACATs) encoded by five genes in Arabidopsis thaliana are targeted either to peroxisomes or cytosol but not to mitochondria. Plant Mol Biol. 2007;63(1):97-108.

49. Wang X, Liang H, Guo D, Guo L, Duan X, Jia Q, Hou X. Integrated analysis of transcriptomic and proteomic data from tree peony (P. ostii) seeds reveals key developmental stages and candidate genes related to oil biosynthesis and fatty acid metabolism. Hortic Res England. 2019;6(1):1-9.

50. Wu B, Ruan C, Han P, Ruan D, Xiong C, Ding J, Liu S. Comparative transcriptomic analysis of high- and low-oil Camellia oleifera reveals a coordinated mechanism for the regulation of upstream and downstream multigenes for high oleic acid accumulation. 3 Biotech. 2019;9(7):257.

51. Wan H, Cui Y, Ding Y, Mei J, Dong H, Zhang W, Wu S, Liang Y, Zhang C, Li J, Xiong Q, Qian W. Time-series analyses of transcriptomes and proteomes reveal molecular networks underlying oil accumulation in Canola. Front Plant Sci. 2017;7:2007.

52. Liao P, Woodfield HK, Harwood JL, Chye M, Scofield S. Comparative transcriptomics analysis of Brassica napus $L$. during seed maturation reveals dynamic changes in gene expression between embryos and seed coats and distinct expression profiles of acyl-coa-binding proteins for lipid accumulation. Plant Cell Physiol. 2019. https://doi.org/10.1093/pcp/pcz169.

53. Hong MJ, Jang YE, Kim DG, Kim JM, Lee MK, Kim JB, Eom SH, Ha BK, Lyu Jl, Kwon SJ. Selection of mutants with high linolenic acid contents and characterization of fatty acid desaturase 2 and 3 genes during seed development in soybean (Glycine max). J Sci Food Agr. 2019;99(12):5384-91.

54. Xie D, Dai Z, Yang Z, Tang Q, Deng C, Xu Y, Wang J, Chen J, Zhao D, Zhang S, Zhang S, Su J. Combined genome-wide association analysis and transcriptome sequencing to identify candidate genes for flax seed fatty acid metabolism. Plant Sci. 2019;286:98-107.

55. Lv J, Zhao F, Feng J, Liu Q, Nan F, Liu X, Xie S. Transcriptomic analysis reveals the mechanism on the response of Chlorococcum sp. GD to glucose concentration in mixotrophic cultivation. Bioresource Technol. 2019;288:121568.

56. Grabherr MG, Haas BJ, Yassour M, Levin JZ, Thompson DA, Amit I, Adiconis X, Fan L, Raychowdhury R, Zeng QD, Chen ZH, Mauceli E, Hacohen N, Gnirke A, Rhind N, di Palma F, Birren BW, Nusbaum C, Lindblad-Toh K, Friedman N, Regev A. Full-length transcriptome assembly from RNA-seq data without a reference genome. Nat Biotechnol. 2011;29(7):130-644.

57. Trapnell C, Williams BA, Pertea G, Mortazavi A, Kwan G, van Baren MJ, Salzberg SL, Wold BJ, Pachter L. Transcript assembly and quantification by RNA-seq reveals unannotated transcripts and isoform switching during cell differentiation. Nat Biotechnol. 2010;28(5):174-511.

58. Robinson MD, McCarthy DJ, Smyth GK. EdgeR: a bioconductor package for differential expression analysis of digital gene expression data. Bioinformatics. 2010;26(1):139-40.

59. Mao X, Cai T, Olyarchuk JG, Wei L. Automated genome annotation and pathway identification using the KEGG orthology (KO) as a controlled vocabulary. Bioinformatics. 2005;21 (19):3787-93.

60. Young MD, Wakefield MJ, Smyth GK, Oshlack A. Gene ontology analysis for RNA-seq: accounting for selection bias. Genome Biol. 2010;11(2):R14

61. Silva TS, Richard N. Visualization and differential analysis of protein expression data using R. New York: Humana Press; 2016.

62. Wang X, Lu P, Luo Z. GMATO: a novel tool for the identification and analysis of microsatellites in large genomes. Bioinformation. 2013;9(10):541-4.

63. Hui W, Yang Y, Wu G, Peng C, Chen X, Zayed MZ. Transcriptome profile analysis reveals the regulation mechanism of floral sex differentiation in Jatropha curcas L. Sci Rep UK. 2017;7(1):16421.

64. Hui W, Wang Y, Chen X, Zayed M, Wu G. Analysis of transcriptional responses of the inflorescence meristems in Jatropha curcas following gibberellin treatment. Int J Mol Sci. 2018;19(2):432.

\section{Publisher's Note}

Springer Nature remains neutral with regard to jurisdictional claims in published maps and institutional affiliations.

Ready to submit your research? Choose BMC and benefit from:

- fast, convenient online submission

- thorough peer review by experienced researchers in your field

- rapid publication on acceptance

- support for research data, including large and complex data types

- gold Open Access which fosters wider collaboration and increased citations

- maximum visibility for your research: over $100 \mathrm{M}$ website views per year

At BMC, research is always in progress.

Learn more biomedcentral.com/submissions 